\title{
Generation of shocked hot regions in black hole magnetosphere
}

\author{
Keigo Fukumura, ${ }^{1}$ Masaaki Takahashi ${ }^{2}$ and Sachiko Tsuruta ${ }^{3}$ \\ ${ }^{1}$ NASA/Goddard Space Flight Center, Code 663, Greenbelt, MD 20771, USA \\ ${ }^{2}$ Department of Physics and Astronomy, Aichi University of Education, Kariya, \\ Aichi 448-8542, Japan \\ ${ }^{3}$ Department of Physics, Montana State University, Bozeman, MT 59717, USA \\ email: fukumura@milkyway.gsfc.nasa.gov, takahasi@phyas.aichi-edu.ac.jp, \\ uphst@gemini.msu.montana.edu
}

\begin{abstract}
We study magnetohydrodynamic (MHD) standing shocks in ingoing plasmas in a black hole (BH) magnetosphere. We find that low or mid latitude (non-equatorial) standing MHD shocks are both physically possible, creating very hot and/or magnetized plasma regions close to the event horizon. We also investigate the effects of the poloidal magnetic field and the BH spin on the properties of shocks and show that both effects can quantitatively affect the MHD shock solutions. MHD shock formation can be a plausible mechanism for creating high energy radiation region above an accretion disk in AGNs.
\end{abstract}

Keywords. Black hole physics - magnetohydrodynamics: MHD - shock waves

\section{Introduction}

In a series of our previous investigations in the context of general relativity (see, e.g., Takahashi et al. 2002, 2006) it has been shown that shock formation in MHD plasmas in-flowing onto a black hole can be a physically plausible mechanism for creating very hot (i.e., $T_{i} \gtrsim 10^{12} \mathrm{~K}$ for ions and $T_{e} \gtrsim 10^{9} \mathrm{~K}$ for electrons) or strongly magnetized plasma regions. These studies were conducted primarily for the equatorial accretion flows. As a natural next step we extended these studies to two dimensional, non-equatorial flows. Specifically, we investigated the polar angle dependence of various shock properties. Nonequatorial shock-heated regions would be attractive as a possible high energy radiation source above an accretion disk in the central engine of active galactic nuclei (AGN). Solid understanding of non-equatorial shock formation can be useful for comparing our theoretical implications with future observations.

\section{Our models, results and conclusion}

We considered the MHD shock formation around a Schwarzschild and slowly-rotating Kerr black hole. The basic equations governing plasmas consist of particle number conservation law, the equation of motion, and the ideal MHD condition. We solved these equations for wide ranges of field-aligned flow parameters, by adopting stationary and axisymmetric ideal MHD accretion flows in Kerr geometry, and applying the general relativistic Rankine-Hugoniot shock conditions in Kerr geometry - particle number conservation, energy conservation, and angular momentum conservation. The background metric is written by the Boyer-Lindquist coordinates. We systematically explored, for 
the first time, general relativistic MHD shock formation by employing the conserved quantities and found the allowed shocked regions.

In order to explore a general trend for allowed MHD shock formation for the vast amount of parameter sets, we sliced the entire parameter space, consisting of all of the parameters (i.e., the total plasma energy $E$, plasma angular momentum $L$, angular velocity of the magnetic field line $\Omega_{F}$, particle flux per magnetic flux $\eta$, dimensionless postshock plasma temperature $\theta_{\mathrm{sh}}$, magnetization parameter $\delta$, and spin parameter $a$ ), by finding the allowed cubic parameter space, consisting of three parameters $(E, L$, and $\Omega_{F}$ ), for given black hole magnetosphere models with the other two parameters $a$ and $\delta$ fixed. Since it is very hard to explore all the parameter space spanned by all of the parameters together, our current approach will greatly simplify and help us better understand a comprehensive picture of the resulting shock solutions. Through our search for possible non-equatorial MHD shocks we find that the allowed shock region is constrained by various physical factors - especially the regularity conditions at the magnetosonic points and the shock condition. Because the mathematical expressions for the regularity conditions for the existence of the Alfven and fast magnetosonic points are not at all simple (see, e.g., Takahashi et al. 2002), the topological appearance of the obtained shock regions is also complicated, but nevertheless we find it very useful. It may be emphasized that our search for the parameter space which allows MHD shock formation is carried out in a systematic manner in such a way that our choice of the parameter sets is not arbitrary. It is consistent, for instance, with the numbers relevant in application to, e.g., the environment of black hole magnetospheres around supermassive black holes in AGN.

Rotation and geometry of the magnetic field are among the primary factors for generating a strong shock. We find that the shocked plasma temperature has a clear anti-correlation with the shocked plasma magnetization. The more strongly magnetized plasma is formed for larger $L \Omega_{F}$. We also show that the energy transport between the fluid and the magnetic field can operate even more effectively across the shock front.

In summary we find that non-equatorial MHD shocks can form and could be a plausible candidate for generating a hot and/or strongly magnetized region over various latitudes in the non-equatorial plane, which could be a powerful high energy radiation source. It may be noted that in reality the actual MHD shock location in the actual astrophysical environment would probably trace a certain trajectory, rather than occupying the whole allowed shock region, because the parameters allowing the shock formation may be unique from case to case.

In various astrophysical objects, high energy activities are often associated with the magnetic fields along which the accreting plasma flows. Our investigation of MHD standing shock formation will be useful for our better understanding of complicated interactions between the plasma and the magnetic field in these situations. In the context of a strong radiation source required for the accretion-powered central engines of objects such as AGNs and GBHCs, the presence of non-equatorial shocks therefore can be attractive as a candidate for such a source.

Further details can be found in Fukumura et al. 2006.

\section{References}

Fukumura, F., Takahashi, M. \& Tsuruta, S. 2006, ApJ, submitted

Takahashi, M., Rilett, D., Fukumura, K. \& Tsuruta, S. 2002, ApJ, 572, 950

Takahashi, M., Goto, J., Fukumura, K., Rillet, D. \& Tsuruta, S. 2006, ApJ, submitted; astro$\mathrm{ph} / 0511217$ 